\title{
Retrieval Attempts Transiently Interfere with Concurrent Encoding of Episodic Memories But Not Vice Versa
}

\author{
Kevin Allan and Roy Allen \\ Cognitive Electrophysiology and Memory Laboratory, College of Life Sciences and Medicine, School of Psychology, University of Aberdeen, Aberdeen AB24 \\ 2UB, United Kingdom
}

In the rodent hippocampus, different phases of each theta activity cycle may be devoted to encoding and retrieval processes. These cycles of $\sim 3-8 \mathrm{~Hz}$ would allow equal processing time for each state and also provide temporal segregation to minimize their mutual interference. We show here that, by controlling the presentation asynchrony between verbal encoding and retrieval cues, theta-resolution $(<100$ $\mathrm{ms}$ ) interference-free shifts between functional states are not expressed in hippocampally dependent, human "episodic" memory. Instead, retrieval attempts selectively and transiently interfere, for $\sim 450 \mathrm{~ms}$, with the encoding of ongoing experiences. Analyses of scalp event-related potentials confirmed that the functional state of the brain during retrieval is largely unperturbed by concurrent encoding and also suggested that encoding impairments may last until a neocortical phase of retrieval can begin. The findings reveal the dynamic properties of interdependent encoding and retrieval functions that contribute to episodic memory in vivo and, moreover, show that, in humans, this form of memory does not operate with either the equality, or the rapidity, intrinsic to the theta model of rodent hippocampal function.

Key words: hippocampus; theta rhythm; episodic memory; encoding; retrieval; event-related potentials

\section{Introduction}

The sharing of neural substrates between encoding and retrieval is a central organizing principle in current memory models ( $\mathrm{Co}$ hen and Eichenbaum, 1993; McLelland et al., 1995; Squire et al., 2004). Here, we examine an unexplored consequence of the principle for human "episodic" memory function in vivo. We investigate whether competition for shared hippocampal substrates produces mutual interference when new memory traces are formed at the same time as attempts are made to retrieve existing traces.

Recent rodent neurophysiological work (Hyman et al., 2003) and computational simulations (Kunec et al., 2005) have begun to elucidate activity dynamics that allow the hippocampus to play a role in both encoding and retrieval. A key idea is that encoding and retrieval activities need temporal segregation to avoid their mutual interference within circuits that interconnect subfields CA1 and CA3 with their entorhinal input/output pathways. Effective temporal segregation could be achieved by synchronizing entorhinal inputs to cyclic fluctuations in hippocampal longterm potentiation (LTP) and recurrent CA3 and CA3-CA1 synaptic activity (Kunec et al., 2005; Rizzuto et al., 2003) [for an alternative model, see Meeter et al. (2004)]. To prevent interference from or with existing hippocampal traces, this model pro-

\footnotetext{
Received April 5, 2005; revised July 8, 2005; accepted July 14, 2005.

This work was supported by British Biotechnology and Biological Sciences Research Council Grant 1/S15294. We thank two anonymous reviewers for their very useful comments on a previous version of this manuscript.

Correspondence should be addressed to Dr. Kevin Allan, Cognitive Electrophysiology and Memory Laboratory, School of Psychology, College of Life Sciences and Medicine, University of Aberdeen, Aberdeen AB24 2UB, UK. E-mail: k.allan@abdn.ac.uk.

D01:10.1523/JNEUROSCI.1304-05.2005

Copyright $\odot 2005$ Society for Neuroscience $\quad 0270-6474 / 05 / 258122-09 \$ 15.00 / 0$
}

poses that formation of a new hippocampal trace is facilitated by the arrival of entorhinal input at CA1 synapses when LTP is maximal and when recurrent CA3 and CA3-CA1 synaptic connections are least active, conditions observed to coincide in vivo and in vitro with peak phases of hippocampal theta (Huerta and Lisman, 1993; Pavlides et al., 1988; Hyman et al., 2003). Conversely, rapid access to existing traces is facilitated if retrieval cue information arrives at CA1 synapses when LTP is weakest, and input from CA3 is strong, conditions that coincide with troughs in hippocampal theta (Hyman et al., 2003).

Temporally segregating encoding and retrieval to different phases of the theta cycle may offset any adaptive disadvantages arising from the dual functional role of the hippocampus. In particular, this occurs by minimizing "hysteresis" effects when shifting from encoding to retrieval states and vice versa. However, if $3-8 \mathrm{~Hz}$ theta rhythms guide its transitions, the hippocampus may not distinguish and independently process entorhinal inputs representing to-be-encoded patterns or retrieval cues that arrive within the $\sim 100 \mathrm{~ms}$ period between consecutive theta peaks and troughs. Here, we performed two experiments, with healthy adult human participants, to discover whether there are, in fact, any limits on temporal segregation between encoding and retrieval in the hippocampally dependent episodic form of memory. Using a novel dual-task paradigm (see Fig. 1), we controlled the stimulus onset asynchrony (SOA) of encoding and retrieval cues to examine how their temporal overlap affects memory, relative to "baseline" conditions in which cues were given in isolation. Experiment 1 combined high-temporal resolution scalp event-related potential (ERP) measures with behavioral performance data to reveal the time course and "pattern" (mutual or unidirectional) of interference effects within the $200 \mathrm{~ms}$ theta period. Experiment 
2 used an extended SOA range, up to $2 \mathrm{~s}$, to reveal the full time course of interference effects.

\section{Materials and Methods}

Participants. In experiment 1, participants were 16 healthy right-handed adults (five males; $17-48$ years of age) recruited from the University of Aberdeen undergraduate student population. In experiment 2, a different sample of 30 right-handed participants ( 11 males; $18-31$ years of age) was recruited from the same student population.

Procedures. Both experiments used the same set of 400 low- to medium-frequency words (Wilding and Rugg, 1997). For auditory presentations, each word was digitized at $22 \mathrm{kHz}$ with 16-bit resolution and then edited so that the start of the stored sound segment corresponded with the onset of the spoken word. All words were recorded in a male (average duration, $660 \mathrm{~ms}$ ) and a female (average duration, $630 \mathrm{~ms}$ ) voice. For visual presentation, words were displayed for $500 \mathrm{~ms}$ at the center of a 17 inch computer monitor in white 24-point Times New Roman font on a black background. Horizontal visual angle was never $>5^{\circ}$ at the viewing distance of $0.57 \mathrm{~m}$, and vertical visual angle was $3^{\circ}$. All stimuli were randomly assigned to conditions before each experimental run and item order was also randomized at each stage.

The three-stage dual-task paradigm (see Fig. 1) was implemented using Presentation Control Language, the scripting language of Presentation (Neurobehavioral Systems, Albany, CA). Presentation allows all stimulus timing uncertainties and inaccuracies deriving from the personal computer (PC) hardware/operating system to be monitored. In experiment 1 , SOA values began at $51 \mathrm{~ms}$ and incremented in nine steps of $17 \mathrm{~ms}$ to a maximum SOA of $204 \mathrm{~ms}$ ( $17 \mathrm{~ms}$ was the finest temporal resolution that we could reliably produce given the refresh rate of our PC monitor). In experiment 2, SOAs began at $51 \mathrm{~ms}$ and incremented in nine steps of $204 \mathrm{~ms}$ to a maximum SOA of $1887 \mathrm{~ms}$. The average inaccuracy in onset times of auditory and visual stimuli across experimental runs was never greater than a few milliseconds.

During stage 1 (see Fig. $1 \mathrm{~A}$ ), participants listened to a series of 80 words, with 40 spoken by a male voice and 40 spoken by a female voice, in a random order. Participants judged whether each word denoted an animate or inanimate object, and no overt response was required. Each trial began with a fixation cross, replaced after $1.0 \mathrm{~s}$ by a blank screen, followed $0.5 \mathrm{~s}$ later by presentation of the auditory stimulus. Four seconds of "encoding" time was given before the fixation cross was presented again for the subsequent trial, giving an intertrial interval of $5.5 \mathrm{~s}$. Stage 1 lasted for $\sim 8 \mathrm{~min}$.

During stage 2 (see Fig. $1 B$ ), which comprised 200 trials in total, participants performed recognition and animacy tasks either singly [no interference ("I-")] or jointly [interference ("I+")]. In each of the $120 \mathrm{I}-$ trials, participants either listened to 1 of 40 studied or 40 unstudied auditory items to decide whether they were presented during stage 1 (giving their decision on 1 of 2 mouse buttons), or they were shown 1 of 40 new items on a monitor screen and judged their animate/inanimate status (no overt response was required). The temporal structure of events on the I- trials was identical to that of the stage 1 trials described above. In each of the 80 stage $2 \mathrm{I}+$ trials, participants attempted both the recognition and the animacy tasks simultaneously. Forty I+ trials contained a stimulus pair comprising an auditory studied item and a new visual item, and the remaining 40 trials contained auditory unstudied items paired with a new visual item.

In both experiments, each of the 10 different SOA values were used in four I + trials, with the encoding or the retrieval cues presented first at each SOA an equal number of times. I+ trial duration varied according to SOA to equate the time available for encoding and retrieval with the $4 \mathrm{~s}$ that is available on I- trials. I+ trial durations therefore ranged in experiment 2 between $5.55 \mathrm{~s}$ (at SOAs of $51 \mathrm{~ms}$ ) and $7.36 \mathrm{~s}$ (at SOAs of 1887 $\mathrm{ms}$ ). In experiment 1 , because SOA values were never $>204 \mathrm{~ms}$, the duration of all $\mathrm{I}+$ trials was fixed at $5.5 \mathrm{~s}$. The order of stage 2 trial types (i.e., I- or I+) was randomized for each participant. Including a short rest after one-half of the trials, on average, stage 2 lasted $\sim 20 \mathrm{~min}$.

In the third and final stage (see Fig. $1 C$ ), a visual recognition task was performed comprising 240 trials, with 120 containing items newly encoded during stage $2(80 \mathrm{I}+$ and $40 \mathrm{I}-$ ) and 120 containing unstudied items. Recognition responses here were also given on mouse buttons. The temporal structure of each stage 3 trial was identical to that of stage 1 trials. Stage 3 lasted an average of $25 \mathrm{~min}$, including a rest pause at the midpoint.

In experiment 1 only, EEG was recorded from $32 \mathrm{Ag}$ - $\mathrm{AgCl}$ electrodes embedded in an elasticized cap (Easy Cap; Falk Minow Services, Herrsching-Breitbrunn, Germany) using an equidistant montage. Recordings were made with an on-line reference to the left mastoid and, subsequently, were algebraically re-referenced to linked left and right mastoids. Vertical and horizontal electro-oculograms (EOGs) were recorded from electrode pairs situated above and to the side of each eye on the outer canthi. EEGs and EOGs were recorded at a rate of $125 \mathrm{~Hz}$ with a bandwidth of $0.03-40 \mathrm{~Hz}$ ( $3 \mathrm{~dB}$ points) and digitized with a 12-bit resolution. Recordings were made continuously throughout all stages of experiment 1 and then epoched off-line, beginning $104 \mathrm{~ms}$ before stimulus onset for a duration of $2048 \mathrm{~ms}$. Epochs containing horizontal or vertical eye movements or eyeblink artifacts were rejected. Epochs were also rejected if analog-to-digital saturation occurred or if baseline drift across the recording epoch exceeded $\pm 50 \mu \mathrm{V}$.

Here, we report analyses conducted on ERPs from each participant, formed to retrieval cues given correct responses during stage 2. ERPs were digitally smoothed $(3 \mathrm{~dB}$ down at $19.4 \mathrm{~Hz})$. Participants did not contribute $<16$ trials in critical conditions (per condition means for the numbers of trials in ERPs are indicated in Fig. 2). ERPs in each condition were quantified by measurement of their mean amplitude (with respect to mean prestimulus baseline) during specific latency regions. The selection of sites for analysis from our montage, and of latency regions, was based on previous ERP literature on episodic retrieval in comparable recognition tasks. Scalp topography analyses used normalized ERP difference scores, according to the method recommended by McCarthy and Wood (1985) and by Wilding (2005). ERP analyses, by ANOVA, used the Greenhouse-Geisser procedure to correct for sphericity violation. All F-ratios from ERP analyses are reported here with their corrected degrees of freedom. The ANOVAs used the factors of interference (I+ vs I-), response type [correct identification of studied items (HIT) vs correction rejection (CR) of unstudied items] and, where indicated, scalp electrode location factors [anterior-posterior, hemiscalp (left vs right), and inferior-superior].

\section{Results}

\section{Experiment 1}

The experiment comprised three distinct stages. In the first stage (Fig. $1 A$ ), a series of auditory samples of single words was played via headphones to each participant. An animacy judgment (living vs nonliving) was performed on each item. All auditory items presented during the first stage were thus encoded with full attention. Participants' ability to discriminate between studied and unstudied auditory items was then tested on single- and dual-task trials in stage 2 of the experiment (Fig. $1 B$ ). Dual-task trials required the performance of the auditory recognition task concurrently with the animacy judgment task on visually presented words. On each dual-task trial, the SOA between the presentation of the auditory retrieval cues and the visual item to be encoded was controlled so that it ranged across 10 different values between 51 and $204 \mathrm{~ms}$ (see Materials and Methods). This SOA manipulation allowed us to examine whether auditory recognition and concurrent visual encoding mutually interfere with one another when their respective cues are delivered in the subtheta time scale. Item presentations were counterbalanced so that auditory and visual cues appeared first equally often across the dual-task trials. Within the constraints of the experimental design used here, this had to be done to ensure that a sufficient numbers of trials were presented at each SOA. The pattern of interference effects described below therefore reflects the temporal proximity of retrieval and encoding processes and not the temporal precedence of either one. Because the dual-task trials were designed to produce interference, performance measures for items presented on these trials were labeled $\mathrm{I}+$.

To detect the presence of interference, it was necessary to obtain contrasting baseline measures of encoding and retrieval performance without interference. These baseline measures were obtained on single-task trials from stage 2 (Fig. $1 B$ ). Speed and accuracy measures of item rec- 
ognition on the single-task trials are labeled Ito denote no interference. The effect of SOA on dual-task recognition was assessed by comparing I+ performance at each of the 10 SOA values against the I- baseline. Interference from concurrent encoding was predicted to manifest as a reduction in recognition levels for $\mathrm{I}+$ items, manifesting at all SOAs within the subtheta time scale. Additional single-task trials were included at stage 2 to obtain baseline measures of visual encoding ability without interference from concurrent auditory recognition attempts. On these trials, visual items were presented for the animacy judgment task. The impact of concurrent retrieval on encoding ability could then be assessed during the third and final stage of the experiment (Fig. 1C). Here, the visually presented items from stage 2 were shown again, along with an equal number of unstudied items, and participants had to make "old/new" recognition judgments.

\section{Behavioral data}

Measures of recognition accuracy and time to respond on the dual-task trials were taken from each participant. I+ performance could then be compared with measures obtained on the single-task trials in which recognition attempts proceeded without any concurrent distraction. The resulting group-average functions for the correct discrimination of studied and unstudied auditory items (i.e., the HIT and CR rates), are shown in Figure 2, $A$ and $B$, along with their corresponding reaction times.

Figure $2 \mathrm{~A}$ shows that, at all SOAs, interference from concurrent encoding has essentially no effect on participants' speed and accuracy at recognizing studied auditory items. Figure $2 B$ depicts a similar lack of interference, at any SOA, on participants' speed and accuracy at identifying unstudied items. Figure $2 \mathrm{C}$ shows group-average recognition performance measures from stage 3 of the experiment, in which participants were asked to judge whether visual items had been studied previously or not during stage 2. The mean CR rate at stage 3 (data not shown in Fig. 2) was $71.0 \%$ $(\mathrm{SD}=13.5)$. Recognition of visual items encoded on I+ trials was initially analyzed separately according to the studied/unstudied status of the concurrently presented auditory item. However, the status of the auditory item was not associated with differences in visual recognition during stage 3 and thus the data in Figure $2 C$ are accordingly collapsed across this factor. It is evident from Figure $2 C$ that the concurrent auditory recognition task, at all SOA values, had an impairing effect on the participants' ability to encode the visual items. The impairment is made manifest in the participants' reduced ability to subsequently recognize I+ visual items compared with the I- visual items.

To establish statistically that interference acted selectively on encoding and left retrieval essentially intact, HIT and CR performance measures at each SOA were subtracted from their corresponding baseline I- rate. The resulting baseline-corrected data were then subjected to ANOVA, using factors of experimental stage (2 vs 3 ) and SOA. ANOVA of the HIT rates gave a main effect of experimental stage, attributable to the reduction in performance relative to baseline found selectively at stage 3 $\left(F_{(1,15)}=10.42 ; p<0.0075\right)$. There was not a main effect or an interaction involving the factor of SOA, reflecting the consistent detriment to performance across all SOA values at stage 3 . ANOVA of the baselinecorrected CR rates did not give rise to any significant main effects or interactions.

These findings demonstrate a clear pattern of interference effects generated when encoding and retrieval are performed in close temporal proximity, but this does not manifest as a mutual impairment to both stages of processing. Instead, retrieval appears to be immune to interference from encoding, to the significant detriment of participants' ability to encode other concurrent elements of their experience.

\section{ERP data}

Neural correlates of retrieval processing under single- and dual-task conditions were compared, revealing evidence of interference-induced changes in the functional state of the brain. These analyses were restricted to well studied ERP modulations called old/new effects (Friedman and Johnson, 2000; Mecklinger, 2000; Rugg and Allan, 2000), comprising differences in ERPs evoked by correct responses to studied and unstudied items (i.e., HITs and CRs, respectively). Endogenous cognitive ERP components, like the old/new effect, predominantly reflect changes in neocortical activity time-locked to the onset of evoking stimuli. Therefore, it is worth emphasizing that the temporal jittering of the encoding and retrieval cues in the dual-task trials ensures that the onset time of the visual stimuli was effectively randomized with respect to the onset time of the auditory recognition cues. This serves to reduce any contamination of the recognition ERPs by encoding-related neural activity. In addition, by restricting the analyses to the differences between HIT and CR ERPs, neural activity common to HITs and CRs in the I+ condition is held constant. Similarly, neural activity common to the HIT and CR ERPs is held constant in the I- condition. "Second-order" contrasts, i.e., between the I- and I+ old/new effects, are thus not confounded by neural activity specific to each condition.

Figure 3 shows the I+ and I- HIT and CR ERPs recorded at a subset of electrode sites situated over the anterior and posterior lateral scalp. For the I+ condition, ERPs comprise data from all dual-task trials, collapsing 

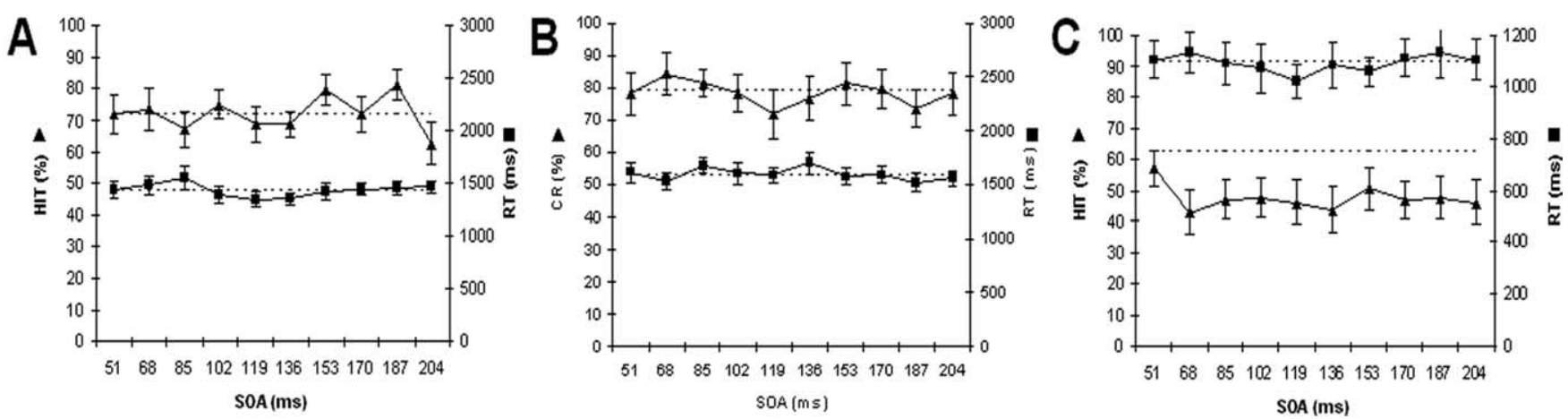

Figure 2. Group average measures of recognition accuracy and reaction times (RTs) from 16 participants in experiment 1. Dashed lines depict baseline (I-) levels, and solid lines depict interference $(I+)$ performance at each SOA. Error bars show SEM \pm 1 . A, Stage 2 correct responses to studied items (HIT) and the associated RT at each SOA. $\mathbf{\Delta}, I+$ HIT percentage; $\mathbf{\square}, I+$ HIT RT. $B$, Stage 2 correct responses to unstudied items [correct rejections (CR)].

, I+ CR RT. C, Stage 3 responses to items encoded during stage $2 . \mathbf{\Delta}, I+$ HIT \%; $\mathbf{\square}$, I+ HIT RT.

across the different SOA values. Within the I- and I+ conditions, differences appear as positive shifts in the HIT ERPs between $\sim 400$ and $1200 \mathrm{~ms}$ after stimulus. Within the I- baseline condition (Fig. 3, top), HIT vs CR ERP differences were not statistically significant during the initial 400-600 ms period, but were reliable between 600 and $800 \mathrm{~ms}$ where, at the selected anterior sites, a main effect of response type was observed $\left(F_{(1,15)}=11.64 ; p<0.005\right)$, reflecting the greater relative positivity of the HIT compared with the CR ERPs. At the posterior sites, ANOVA of 600-800 ms mean amplitude I- data also gave a main effect of response type $\left(F_{(1,15)}=7.80 ; p<0.025\right)$, reflecting the HIT ERP positivity as well as a trend within the interaction between response type and hemiscalp $\left(F_{(1,15)}=3.78 ; p=0.071\right)$, attributable to relatively larger HIT positivity over the posterior left hemiscalp.

In the I+ interference condition (Fig. 3, bottom), ANOVA of the 400-600 ms mean amplitude data gave, at the selected anterior sites, a trend for a response type by hemiscalp interaction $\left(F_{(1,15)}=3.86 ; p=\right.$ $0.069)$, reflecting relatively larger positivity over the left anterior hemiscalp. By 600-800 ms, ANOVA of the I+ HIT vs CR ERPs at anterior sites gave significant main effects of response type, reflecting HIT ERP positivity $\left(F_{(1,15)}=10.54 ; p=0.005\right)$, and also a more robust condition by hemiscalp interaction $\left(F_{(1,15)}=4.40 ; p=0.053\right)$, reflecting the greater amplitude of the positivity over the left compared with the right anterior hemiscalp. During the same interval at the posterior sites, a main effect of response type $\left(F_{(1,15)}=16.05 ; p<0.001\right)$ was observed, attributable to the relative positivity of the HIT compared with the CR ERPs.

In both the I- and the I+ conditions, ANOVAs of mean amplitude data from 800 to $1000 \mathrm{~ms}$ gave main effects of response type [I- anterior $\left(F_{(1,15)}=7.37 ; p<0.025\right), \mathrm{I}-$ posterior $\left(F_{(1,15)}=8.06 ; p<0.025\right)$, and I + posterior $\left.\left(F_{(1,15)}=12.11 ; p<0.005\right)\right]$, but no additional interactions involving the factors of condition and hemiscalp. The HIT and CR ERPs in both conditions do not differ from $\sim 1 \mathrm{~s}$ onward to the end of the epoch at $1944 \mathrm{~ms}$ after stimulus. Finally, direct contrasts between the mean amplitudes of the I- and I+ old/new effects during their entire time course failed to give rise to any main effects or interactions involving the factor of interference condition.

The scalp topographies of the old/new effects during 600-800 ms (Fig. 4) show differences in their left-sided maxima that are consistent with the different loci of the response type by hemiscalp interactions reported above. An additional set of scalp distribution analyses was performed to determine whether there was any evidence of differential neural generator activity in the dual- versus the single-task condition, indicating a qualitative functional change in retrieval processing that might accompany a shift in participants' retrieval strategy. For these distributional analyses, we first formed subtraction waves representing the difference between the HIT and CR ERPs for each condition at each electrode in the subset of anterior and posterior sites, which were then normalized by rescaling (see Materials and Methods). The ANOVA used the factors epoch $(400-600,600-800$, and $800-1000 \mathrm{~ms})$, condition (I- vs I+), anterior-posterior location, hemiscalp, and site. The ANOVA did not reveal any effects of interference condition on topography but did give a three-way interaction between the factors epoch, hemiscalp, and site $\left(F_{(3.1,47.0)}=3.23 ; p<0.05\right)$, reflecting the absence of the left-sided foci from each effect during the final $800-1000 \mathrm{~ms}$ interval. This interpretation was confirmed by subsidiary ANOVAs during each of the $400-600$, $600-800$, and $800-1000 \mathrm{~ms}$ periods, which only gave rise to significant main effects of hemiscalp during the first two intervals $\left(F_{(1,15)}=5.83\right.$, $p<0.05 ; F_{(1,15)}=10.38, p<0.01$, respectively).

\section{Experiment 2}

Experiment 1 demonstrated a consistent impairment to encoding from concurrent retrieval attempts that existed throughout the $<200 \mathrm{~ms} \mathrm{SOA}$ range. In experiment 2, our aim was to replicate and extend the behavioral findings of experiment 1 by mapping out the full time course of this interference effect. The experiment used a larger sample of participants and an extended range of 10 SOAs in 204 ms increments from 51 to 1887 ms (for details, see Materials and Methods). This SOA range mirrors the $\sim 2 \mathrm{~s}$ time scale of the ERP data and therefore allows us to determine whether any temporal correspondences exist between the time courses of the stage 2 ERP old/new effects and the behavioral interference effects that are subsequently manifested during stage 3 .

Figure 5 depicts the results from experiment 2, which appear to provide a good replication of the behavioral findings from experiment 1 . Stage 2 HIT and CR rates and their reaction times do not appear to differ at any SOA value between the It and I- conditions (Fig. $5 A, B$ ). As in experiment 1 , recognition accuracy and reactions times did not differ significantly at any SOA between the two classes of I+ HIT items at stage 3; therefore, responses were again collapsed together to form a single stage $3 \mathrm{I}+$ condition, shown in Figure $5 C$. As is clear from Figure $5 C$, stage 3 impairments in recognition I+ HIT rates, compared with their I- baseline, were dependent on the SOA at which I+ items were presented when first encountered. The mean CR rate from stage 3 (data not shown in Fig. 5) was $68.5 \%(\mathrm{SD}=15.0)$.

As in experiment 1, baseline-corrected HIT rate data from stages 2 and 3 were analyzed first by ANOVA using the factors phase and SOA. The ANOVA gave a main effect of phase, resulting from the reduced performance, relative to baseline in stage $3\left(F_{(1,29)}=24.34 ; p<0.001\right)$ and a significant interaction between phase and SOA $\left(F_{(1,9)}=2.53 ; p<0.01\right)$. To follow up on the interaction, individual one-tailed paired sample $t$ tests were performed to compare HIT rates on stage 3 items encoded at each SOA, which gave significant results only at SOAs of $51 \mathrm{~ms}\left(t_{(29)}=\right.$ $3.01 ; p=0.005), 255 \mathrm{~ms}\left(t_{(29)}=5.80 ; p<0.001\right), 459 \mathrm{~ms}\left(t_{(29)}=4.37\right.$; $p<0.001)$, and $663 \mathrm{~ms}\left(t_{(29)}=2.58 ; p<0.025\right)$. Interference effects disappear from $867 \mathrm{~ms}$ onward [although they briefly reemerge at 1683 $\left.\mathrm{ms}\left(t_{(29)}=2.43 ; p<0.025\right)\right]$. However, application of the conservative Bonferroni-type correction for these 10 multiple comparisons restricts the interval of significant interference effects to between 51 and $459 \mathrm{~ms}$. Analysis of the baseline-corrected HIT reaction time data did not give rise to any significant main effects or interactions involving the factors stage or SOA. SOA also failed to affect the baseline-corrected CR rates from stage 2 . 


\section{Discussion}

Using a retrieval cue transiently impairs the ability to encode other concurrently presented items, as revealed by reductions in the ensuing recognition rates for these items compared with items presented under single-task conditions (Fig. 2C). By manipulating the asynchrony of encoding and retrieval cues, we showed that encoding was impaired at SOAs between 51 and $459 \mathrm{~ms}$ but began to improve at $663 \mathrm{~ms}$ SOA and had returned to baseline levels by $867 \mathrm{~ms}$ SOA (Fig. 5C). Encoding was impaired to the same degree throughout the 51-459 ms interval and was also equivalent regardless of whether retrieval was cued with a previously studied or an unstudied auditory item. Encoding, however, had no discernible behavioral effect on concurrent retrieval throughout the entire 51-1887 ms SOA range used across the present two experiments (Figs. $2 A, B, 5 A, B$ ). It is important to note that, in both experiments, stimulus presentation order was counterbalanced so that encoding and retrieval cues appeared first equally as often. Hence, the encoding impairments do not occur simply because the retrieval cues temporally preceded other elements of an episode. Instead, these impairments are generated by close temporal proximity between a retrieval cue and another element of the ongoing event in which the cue is presented.

Although concurrent encoding had no demonstrable behavioral effect on recognition, there could still have been underlying changes in our participants' retrieval strategy as an adaptation to dual tasking. Hence, we used ERPs to covertly indicate whether or not successful recognition judgments were made on different functional bases under single- and dual-task conditions. Neural activity associated with successful recognition was revealed by contrasting ERPs in the HIT and CR conditions, which gave statistically robust differences during the 600-1000 ms interval after the presentation of retrieval cues. HIT ERPs during this interval exhibited positive-going shifts that were initially pronounced over the left hemiscalp. However, the exact foci of these early left asymmetries were slightly different in the Iand $\mathrm{I}+$ conditions. Recognition during the single-task I- trials was associated with a temporoparietal left hemiscalp effect (Fig. $4 A$ ), whereas recognition during the dual-task $\mathrm{I}+$ trials produced a more anteriorly distributed left hemiscalp effect [which began to manifest earlier (400-600 $\mathrm{ms}$ ) than was the case in the I- condition] (Fig. 4B). However, no differences were
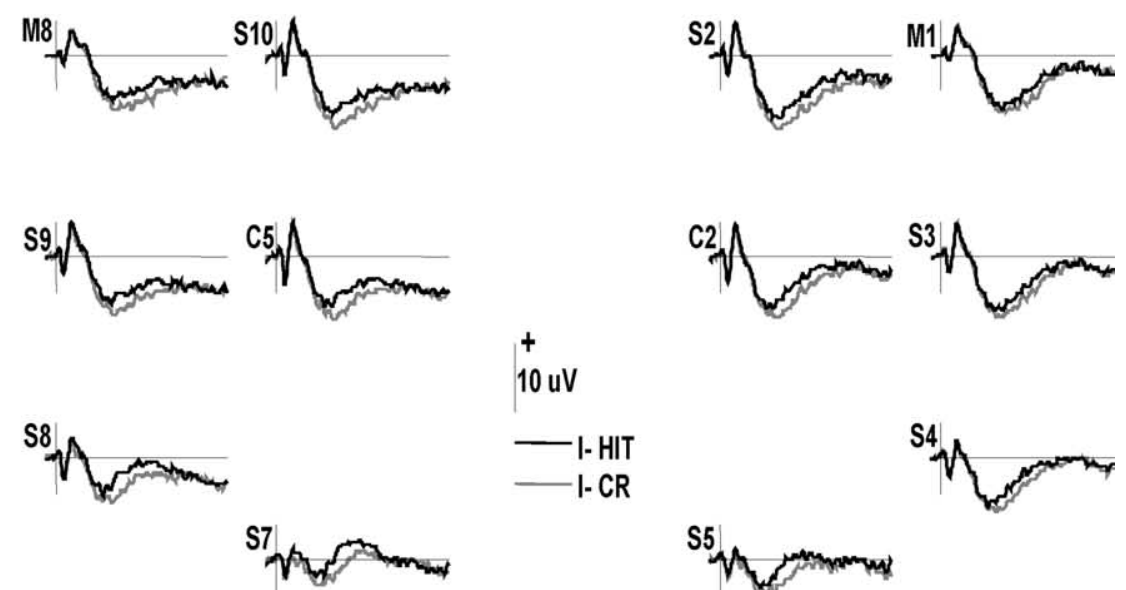

$10 \mathrm{uV}$
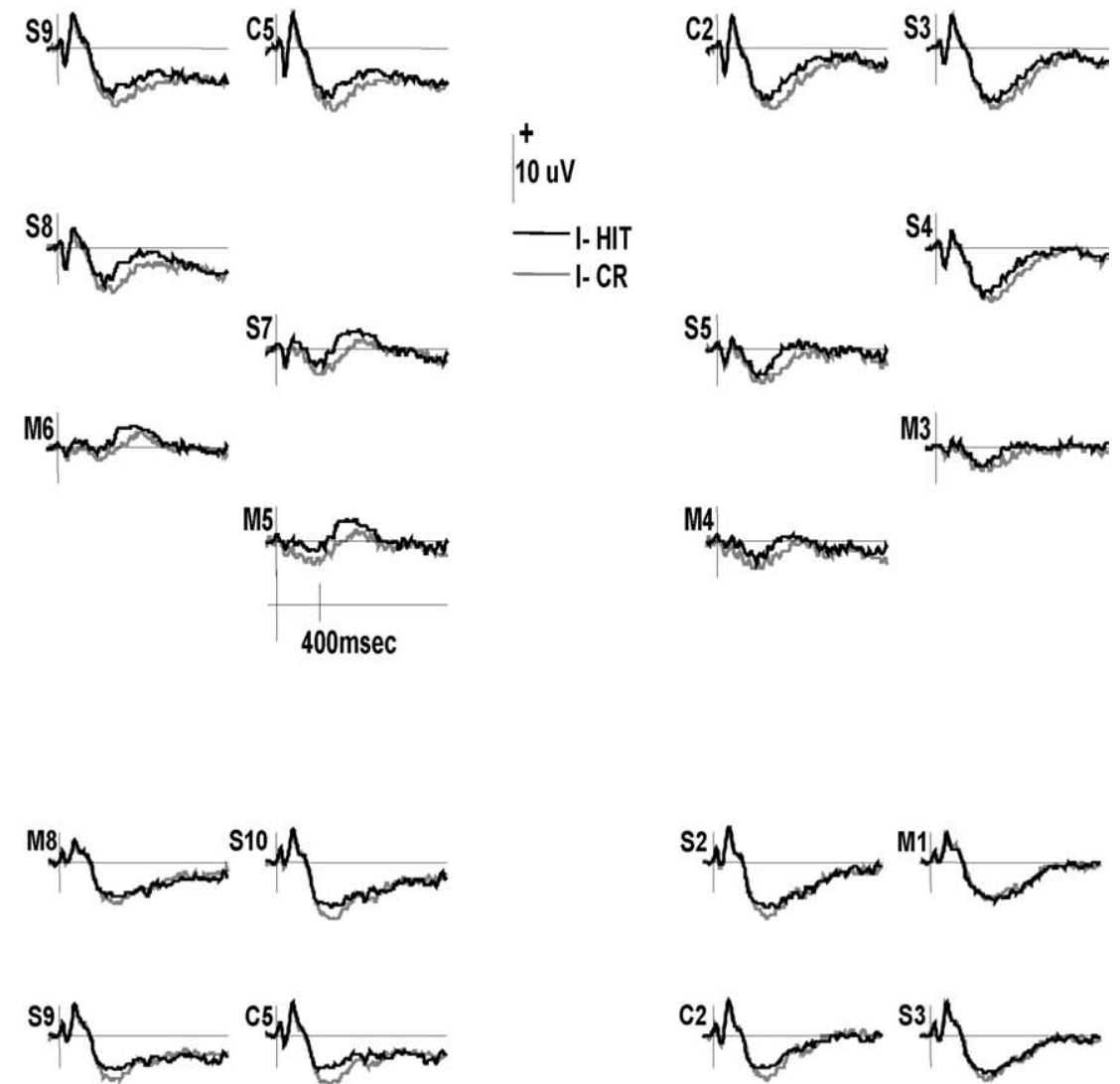

+
$10 \mathrm{uV}$
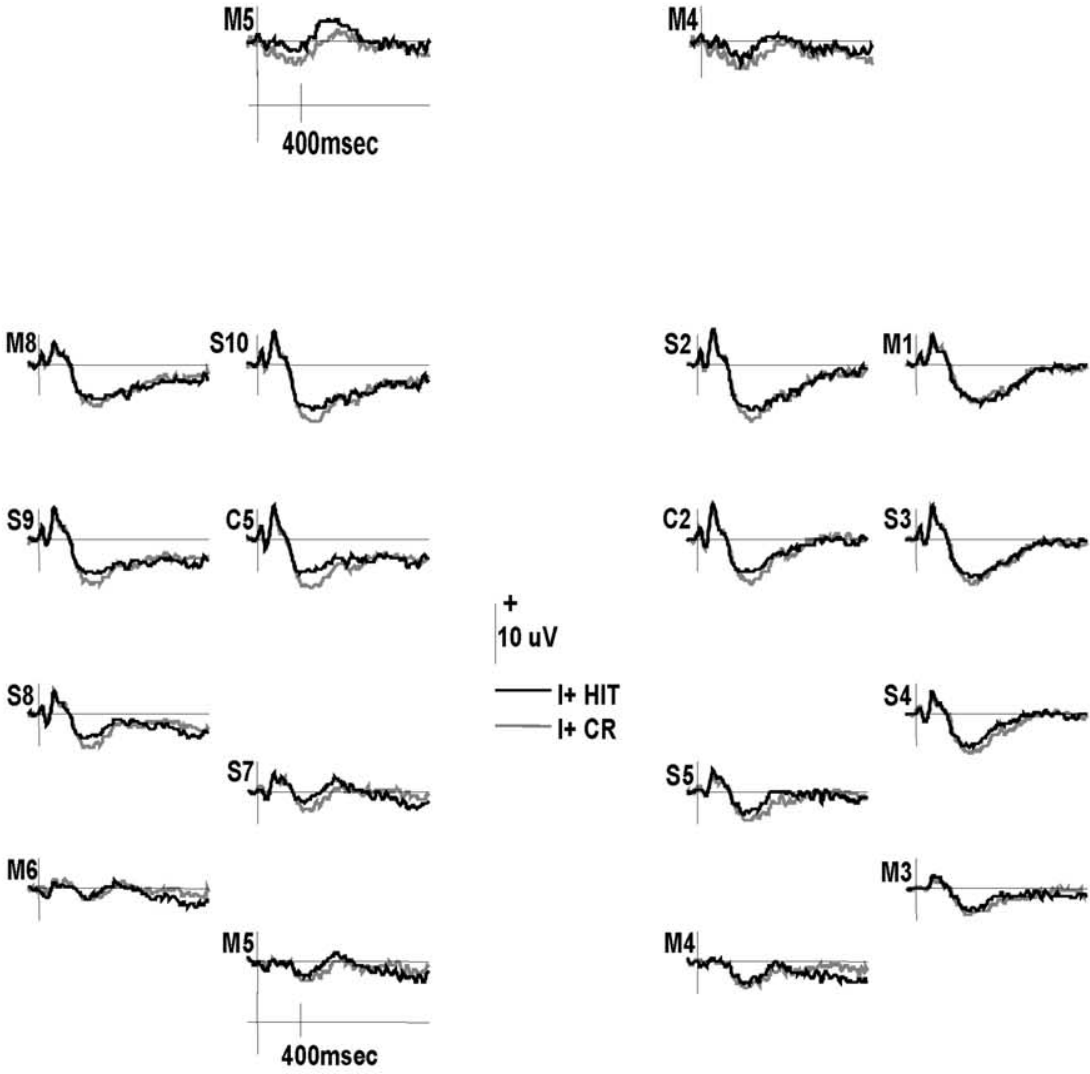

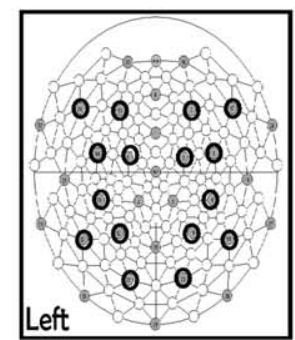

Figure 3. Group-average recognition ERPs from the 16 participants in experiment 1, evoked during stage 2 by studied and unstudied items given correct responses [HIT and correct rejection (CR), respectively]. Top, Single-task I- . The mean number of trials per participant in the HIT and CR ERPs was 25.5 (range, 18 -34) and 27.8 (range, 17-38), respectively. Bottom, Dual-task I+. The mean number of trials per participant in the HIT and CR ERPs was 25.8 (range, 16-33) and 28.4 (range, 17-35), respectively. The electrode labels refer to their position within the concentric ring montage centered on the location of the Czelectrode of the 10/20 system (Jasper, 1958). Cn, Central; Sn, superior; $\mathrm{Mn}$, midlateral. $n=$ the clockwise ordinal position beginning with the electrode that lies on the sagittal plane through $\mathrm{C}$. Electrode scalp locations are ringed on the inset diagram at the bottom. 
A
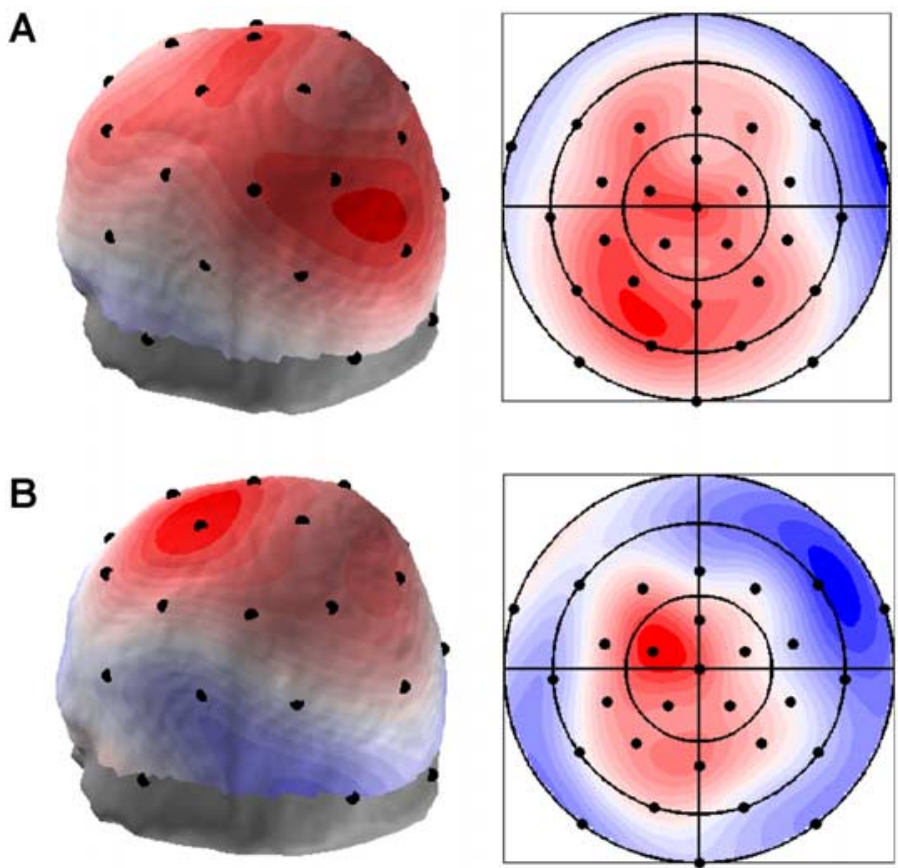

Figure 4. Two-dimensional and three-dimensional topographic maps of the difference between HIT and CRERPs (old/new ERP effect), from the 16 participants in experiment 1, representing the $600-800 \mathrm{~ms}$ interval after the onset of retrieval cues during stage 2 . The bar on the right depicts the microvolt range of the effect, with red shades indicating areas of relative positivity in the HIT compared with the CR ERPs. A, Single-task I-. B, Dual-task I+.

detected in the underlying neural generators of the I- and I+ old/new effects, suggesting that interference from concurrent encoding did not qualitatively alter the functional basis on which correct recognition judgments were made.

Our experiments demonstrate that segregation between encoding and retrieval processes in human episodic memory breaks down when they are triggered within $\sim 450 \mathrm{~ms}$ of each other, well above the $\sim 100 \mathrm{~ms}$ resolution of theta-linked hippocampal cycles (Kunec et al., 2005). Our findings therefore imply that the ability of the human brain to avoid interference between each processing stage does not reflect segregation of encoding and retrieval to different phases of a theta cycle. We obviously do not claim to have demonstrated a failure to conserve such cycles. Instead, we showed that the functional architecture of episodic memory has slower segregation capabilities than we would expect if theta-linked cycles were the sole determining factor. Our findings are therefore more in agreement with models that posit slower-acting shifts between hippocampal encoding and retrieval states (Lisman and Otmakhova, 2001; Meeter et al., 2004; Lisman and Grace, 2005). An additional consequence of the theta-linked encoding/retrieval model is that cyclic changes in physiological state allow the hippocampus to efficiently serve both functions, without prioritizing one at the expense of the other (Hasselmo et al., 2002; Kunec et al., 2005). Our findings suggest, however, that such equality may not exist in human episodic memory, which appears to be able to prioritize retrieval processing at the expense of concurrent encoding, even when the retrieval task involves relatively simple old/new recognition judgments.

Evidence in humans for the prioritization of retrieval over other concurrent cognitive tasks has been obtained in previous dual-task studies (Craik et al., 2000; Naveh-Benjamin et al., 2000) (cf. Fernandes and Moscovitch, 2002; Fernandes et al., 2004), and our experiments here extend this work in two specific ways: first,
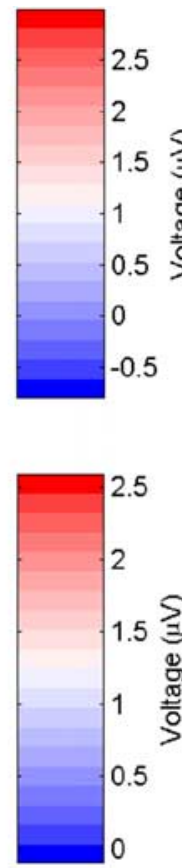

by demonstrating the impact retrieval prioritization has on concurrent episodic encoding and second, by revealing the fine-grained temporal properties of the interdependency between encoding and retrieval processes. Straightforward adaptations to instructions and stimulus materials will allow future use of the three-stage dual task to explore the dynamics of encoding/retrieval interactions with considerable flexibility and experimental control. Moreover, the fine-grained temporal information obtained may be compared with detailed knowledge on the time course of neuronal activity from studies of memory function in nonhuman animals and from depth electrode recordings in human neuropsychological patients, as we show below.

The apparent prioritization of retrieval processing was a transient phenomenon that appeared to end when changes in neural activity associated with successful retrieval began to manifest in ERP recordings. We hypothesize that this temporal correspondence is a direct expression of dynamic resource sharing between encoding and retrieval. A critical question, then, concerns what happens at $\sim 600-800 \mathrm{~ms}$ SOAs such that encoding becomes free of interference. It has been repeatedly demonstrated, in previous intracranial electrode recording work with human epileptic patients, that the $\sim 500 \mathrm{~ms}$ period after stimulus onset is critical to effective medial temporal lobe encoding processes (Halgren et al., 1985; Heit et al., 1988, 1990; Fernandez et al., 1999, 2002; Fell et al., 2002; Paller and McCarthy, 2002) [for congruent findings in monkeys, see Ringo (1995) and Messinger et al. (2001)]. If hippocampal activity within this interval does play a crucial role in encoding, any disruption of that activity should have a negative impact on subsequent memory, whereas disruption outwith the interval should not (all else being equal). Which is essentially what we observed here in experiment 2.

Impairments to subsequent recognition ability attributable to disruption of hippocampal encoding activity have been reported in a recent experiment by Coleshill et al. (2004) that used subepileptogenic electrical stimulation (ES) applied directly to the hippocampus in human epilepsy patients. ES was time locked to the presentation of visual stimuli during the encoding phase of a recognition memory task similar to the one we used in the present experiments, resulting in recognition impairments for such items compared with items presented for encoding without concurrent ES. The effect of varying the SOA between ES and encoding stimuli was not explored; however, our own findings suggest that this could be a promising approach for future studies. In particular, our findings suggest that, in long-term recognition tasks, i.e., ones that do not engage a capacity-limited short-term working memory, the effect of ES may be dependent on its asynchrony with to-be-encoded stimuli. If proven to be correct, this hypothesis would provide substantial support for the existence of a critical encoding interval in hippocampal activity in vivo that immediately follows the onset of a stimulus. Similarly, the effect of SOA between hippocampal ES and the presentation of retrieval cues, 

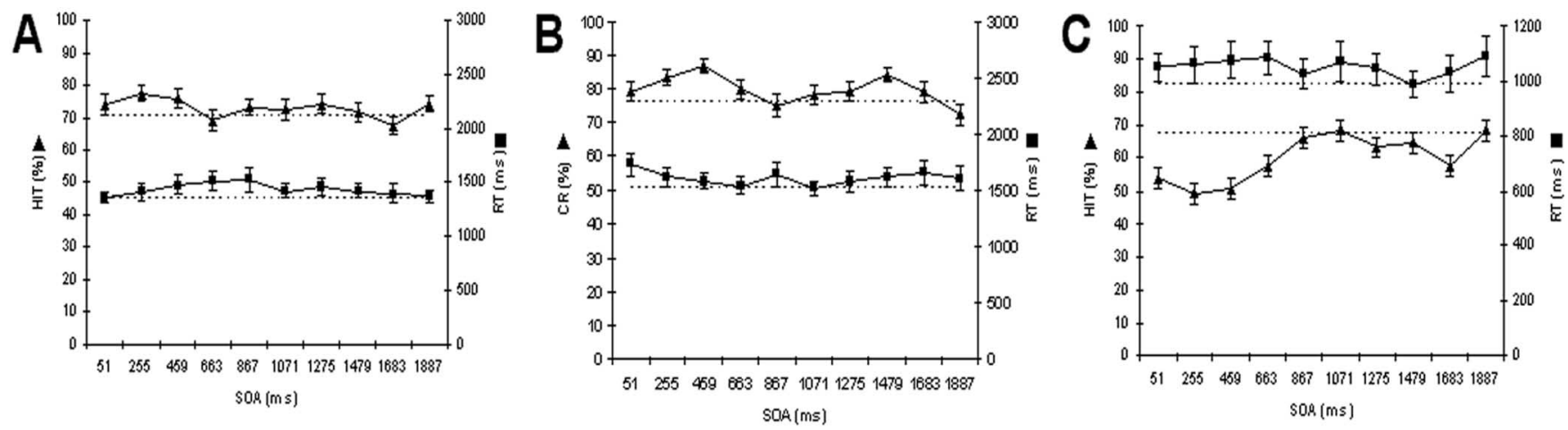

Figure 5. Group average recognition accuracy and reaction times from 30 participants in experiment 2 ( $\boldsymbol{A}-\boldsymbol{C}$ as per the legend to Fig. 1).

using the present three-phase dual-task paradigm, could reveal much about the dynamic interdependence of encoding and retrieval activities within the hippocampus. Sobotka et al. (2005) also showed recently that delay-interval ES directly in the monkey hippocampus, persisting for $125-250 \mathrm{~ms}$ but delivered $1 \mathrm{~s}$ after onset of the sample stimulus, disrupts recognition in a short-term delayed matching-to-sample task. Their findings, in conjunction with our own and those of Coleshill et al. (2004), suggest that the hippocampus may be differentially engaged, or controlled, in long-term versus short-term (working) memory tasks.

The dynamics of hippocampal activity just discussed imply the existence of a critical window of approximately one-half of a second that immediately follows the onset of a stimulus to be encoded. Evidence on the precise timing of hippocampal retrieval-related activity and associated cortical changes in humans (Halgren et al., 1985; Heit et al., 1988; Paller and McCarthy, 2002) and in nonhuman animals (Naya et al., 2003) suggests that a similar critical interval of hippocampal activity follows the onset of a retrieval cue. For example, Naya et al. (2003) examined the informational content of activity in monkey inferotemporal area TE neurons during the delay interval in a paired-associate retrieval task. They observed that changes in neuronal activity in area TE began at $\sim 600 \mathrm{~ms}$ after the onset of cues that triggered the retrieval of previously learned associates from long-term memory. Naya et al. (2003) concluded that this delay period TE activation most likely reflects signaling from the hippocampus that is fed to TE via inferotemporal area 36. In the present experiments, we observed a similar onset time, of $\sim 600 \mathrm{~ms}$, in the ERP old/new effects that were associated with successful retrieval. Furthermore, the onset time of the ERP old/new effects coincided remarkably well with the offset of interference effects on encoding.

We are led to the conclusion that the return of encoding to baseline levels that occurs when the ERP old/new effects emerge may signal the end of a prioritized hippocampal contribution to retrieval. This interpretation is consistent with current views on the functional significance of the ERP old/new effects, which link them to changes in neocortical activity triggered by the output from hippocampal pattern completion operations acting on retrieval cues (Wilding and Rugg, 1997; Allan et al., 1998; Mecklinger, 2000; Rugg and Yonelinas, 2003). This account adheres to the consensual view that distributed neocortical sites store the representational content that underlies the experiential quality of recollection, whereas the hippocampus, in effect, indexes the pattern of neocortical activity specific to each episode (Cohen and Eichenbaum, 1993; McLelland et al., 1995; Nadel and Mosco- vitch, 1997; Moscovitch and Nadel, 1998; Eichenbaum, 2000; Gilboa et al., 2004; Miyashita, 2004; Squire et al., 2004). The posterior left temporoparietal old/new component observed here in the single-task I- condition (Fig. 3, top) has particularly clear empirically established links to processing contingent on the reactivation of episodic traces (Wilding and Rugg, 1997; Allan and Rugg, 1998; Rugg and Allan, 2000; Donaldson et al., 2003; Paller et al., 2003; Curran, 2004). The functional status of the more anterior foci of the dual-task I+ old/new effect (Fig. 3, bottom) is less well established; however, it has been linked to recognition judgments based on item "familiarity" (Rugg et al., 1998; Friedman and Johnson, 2000; Mecklinger, 2000; Tsivilis et al., 2001; Donaldson et al., 2003), a more automatic form of memory that could be used when attentional resources are insufficient to support "full-blown" recollection (Yonelinas, 2002). The presence of the anterior component in the dual-task I+ condition thus fits well with, and provides additional support for, the familiarity account.

Klimesch et al. $(2000,2005)$ proposed recently that oscillatory neocortical changes in $\delta$-band $(2-4 \mathrm{~Hz})$ activity may generate temporoparietal old/new effects. Such changes are sustained in time from the onset of retrieval cues, occur in response to new (unstudied) and studied cues (more so the latter), peak at $\sim 600-800 \mathrm{~ms}$ after stimulus, and coexist with a transient change in theta-band power that occurs $\sim 200-400 \mathrm{~ms}$ after stimulus. Based on this pattern of findings, Klimesch et al. (2005) suggested that the $\delta$-band changes are associated with processes that detect the reactivation of a memory trace. In contrast, Klimesch et al. $(2000,2005)$ associated the more transient theta-band changes with neocortical trace reactivation induced via re-entrant corticohippocampal connectivity. Our present findings fit very well within this framework. As noted in Results, we found that studied and unstudied recognition cues produced equivalent interference effects on encoding, which persist until ERP old/new effects begin to manifest at $\sim 600 \mathrm{~ms}$ after stimulus. The $\delta$-frequency range of $\sim 2-4$ $\mathrm{Hz}$ has a sinusoidal period of $0.25-0.5 \mathrm{~s}$. Hence, the duration of the interference effect is consistent with a process that manifests on a $\delta$-linked time scale rather than the more rapid theta cycle. Our findings therefore suggest that episodic encoding is disrupted by the sustained "capture" of this $\delta$-band activity in the service of retrieval, e.g., by a "reset" mechanism (cf. Rizzuto et al., 2003).

In sum, by manipulating the asynchrony of presentation times for encoding and retrieval cues, we showed that a transient interference could be created that selectively impairs encoding and leaves retrieval performance intact. The time course of the interfer- 
ence effect reveals limits on the human ability to efficiently segregate encoding and retrieval processes so that they do not interfere with one another. This ability was found to operate at a resolution slower than that possible for theta-linked changes in a physiological state that were proposed recently as a mechanism to keep encoding and retrieval separate within the rodent hippocampus. Instead, the interference from retrieval on encoding appears to last about as long as it takes to reactivate the neocortical memory trace of a target episode, as indicated by the time course of putative ERP correlates of this reactivation process. These findings indicate that, within the interval preceding the onset of the ERP old/new effects, hippocampal functioning may be targeted more toward the processing of retrieval cues than on the encoding of other concurrent elements of experience. This may occur if neocortical processes are engaged to sustain and focus attention on retrieval cues, drawing these resources away from other concurrent elements of experience. As a result, memory is significantly impaired for experiences that take place during the immediate peri-retrieval period.

\section{References}

Allan K, Rugg MD (1998) Neural correlates of cued-recall with and without retrieval of source memory. NeuroReport 9:3463-3466.

Allan K, Wilding EL, Rugg MD (1998) Electrophysiological evidence for dissociable processes contributing to recollection. Acta Psychol (Amst) 98:231-252.

Cohen NJ, Eichenbaum H (1993) Memory, amnesia and the hippocampal system. Cambridge, MA: MIT.

Coleshill SG, Binnie CD, Morris RG, Alarcon G, van Emde Boas W, Velis DN, Simmons A, Polkey CE, van Veelen CW, van Rijen PC (2004) Materialspecific recognition memory deficits elicited by unilateral hippocampal electrical stimulation. J Neurosci 24:1612-1616.

Craik FI, Naveh-Benjamin M, Ishaik G, Anderson ND (2000) Divided attention during encoding and retrieval: differential control effects? J Exp Psychol Learn Mem Cogn 26:1744-1749.

Curran T (2004) Effects of attention and confidence on the hypothesised ERP correlates of recollection and familiarity. Neuropsychologia 42:1088-1106.

Donaldson DI, Wilding EL, Allan K (2003) Fractionating retrieval from episodic memory using event-related potentials. In: The cognitive neuroscience of memory: episodic encoding and retrieval (Parker AE, Wilding EL, Bussey TJ, eds), pp 39-58. Hove, UK: Psychology.

Eichenbaum H (2000) A cortical-hippocampal system for declarative memory. Nat Rev Neurosci 1:41-50.

Fell J, Klaver P, Elger CE, Fernandez G (2002) The interaction of rhinal cortex and hippocampus in human declarative memory formation. Rev Neurosci 13:299-312.

Fernandes MA, Moscovitch M (2002) Factors modulating the effect of divided attention during retrieval of words. Mem Cognit 30:731-744.

Fernandes MA, Davidson PS, Glisky EL, Moscovitch MA (2004) Contribution of frontal lobe and temporal lobe function to memory interference from divided attention at retrieval. Neuropsychology 18:514-525.

Fernandez G, Effern A, Grunwald T, Pezer N, Lehnertz K, Dumpelmann M, Van Roost D, Elger CE (1999) Real-time tracking of memory formation in the human rhinal cortex and hippocampus. Science 285:1582-1585.

Fernandez G, Klaver P, Fell J, Grunvald T, Elger CE (2002) Human declarative memory formation: segregating rhinal and hippocampal contributions. Hippocampus 12:514-519.

Friedman D, Johnson RJ (2000) Event-related potential studies of episodic memory encoding and retrieval: a selective review. Microsc Res Tech 51:6-28.

Gilboa A, Winocur G, Grady CL, Hevenor SJ, Moscovitch M (2004) Remembering our past: functional neuroanatomy of recollection of recent and very remote personal events. Cereb Cortex 11:1214-1225.

Halgren E, Wilson CL, Stapleton JM (1985) Human medial temporal lobe stimulation disrupts both formation and retrieval of recent memories. Brain Cogn 4:287-295.
Hasselmo ME, Bodelon C, Wyble BP (2002) A proposed function for hippocampal theta rhythm: separate phases of encoding and retrieval of prior learning. Neural Comput 14:793-817.

Heit G, Smith ME, Halgren E (1988) Neural encoding of individual words and faces by the human hippocampus and amygdala. Nature 333: 773-775.

Heit G, Smith ME, Halgren E (1990) Neuronal activity in the human medial temporal lobe during recognition memory. Brain 113:1093-1112.

Huerta PT, Lisman JE (1993) Heightened synaptic plasticity of hippocampal CA1 neurons during a cholinergically induced rhythmic state. Nature 364:723-725.

Hyman J, Wyble B, Goyal V, Rossi C, Hasselmo ME (2003) Stimulation in the hippocampal region CA1 in behaving rats yields LTP when delivered to the peak of theta and LTD when delivered to the trough. J Neurosci 23:11725-11731.

Jasper H (1958) The 10-20 system of the international federation. Electroencephalogr Clin Neurophysiol 10:271-375.

Klimesch W, Doppelmayr M, Schwaiger J, Winkler T, Gruber W (2000) Theta oscillations and the ERP old/new effect: independent phenomena? Clin Neurophysiol 111:781-793.

Klimesch W, Hanslmayr S, Sauseng P, Gruber W, Brozinsky CJ, Kroll NEA, Yonelinas AP, Doppelmayr M (2005) Oscillatory EEG correlates of episodic trace decay. Cereb Cortex, in press.

Kunec S, Hasselmo ME, Kopell N (2005) Encoding and retrieval in the CA3 region of the hippocampus: a model of theta-phase separation. J Neurophysiol 94:70-82.

Lisman JE, Grace AA (2005) The hippocampal-VTA loop: controlling the entry of information into long-term memory. Neuron 46:703-713.

Lisman JE, Otmakhova NA (2001) Storage, recall, and novelty detection of sequences by the hippocampus: elaborating on the SOCRATIC model to account for normal and aberrant effects of dopamine. Hippocampus 11:551-568.

McCarthy G, Wood CC (1985) Scalp distributions of event-related potentials: an ambiguity associated with analysis of variance models. Electroencephalogr Clin Neurophysiol 62:203-208.

McLelland JL, McNaughton BL, O’Reilly RC (1995) Why are there complementary learning systems in the hippocampus and neocortex: insights from the successes and failures of connectionist models of learning and memory. Psychol Rev 102:419-457.

Mecklinger A (2000) Interfacing mind and brain: a neurocognitive model of recognition memory. Psychophysiology 37:565-582.

Meeter M, Murre JMJ, Talamini LM (2004) Mode shifting between storage and recall based on novelty detection in oscillating hippocampal circuits. Hippocampus 14:722-741.

Messinger A, Squire LR, Zola SM, Albright TD (2001) Neuronal representations of stimulus associations develop in the temporal lobe during learning. Proc Natl Acad Sci USA 98:12239-12244.

Miyashita Y (2004) Cognitive memory: cellular and network machineries and their top-down control. Science 306:435-440.

Moscovitch M, Nadel L (1998) Consolidation and the hippocampal complex revisited: in defence of the multiple trace model. Curr Opin Neurobiol 8:297-300.

Nadel L, Moscovitch M (1997) Memory consolidation, retrograde amnesia and the hippocampal complex. Curr Opin Neurobiol 7:217-227.

Naveh-Benjamin M, Craik FI, Gavrilescu D, Anderson ND (2000) Asymmetry between encoding and retrieval processes: evidence from divided attention and a calibration analysis. Mem Cognit 28:965-976.

Naya Y, Yoshida M, Takeda M, Fujimichi R, Miyashita Y (2003) Delay period activities in two subdivisions of monkey inferotemporal cortex during pair association memory task. Eur J Neurosci 18:2915-2918.

Paller KA, McCarthy G (2002) Field potentials in the human hippocampus during the encoding and recognition of visual stimuli. Hippocampus 12:415-420.

Paller KA, Hutson CA, Miller BB, Boehm SG (2003) Neural manifestations of memory with and without awareness. Neuron 38:507-516.

Pavlides C, Greenstein YJ, Grudman M, Winson J (1988) Long-term potentiation in the dentate gyrus is induced preferentially on the positive phase of theta-rhythm. Brain Res 439:383-387.

Ringo JL (1995) Brevity of processing in a mnemonic task. J Neurophysiol 73:1712-1715. 
Rizzuto DS, Madsen JR, Bromfield EB, Schulze-Bonhage A, Seelig D, Aschenbrenner-Scheibe R, Kahana MJ (2003) Reset of human neocortical oscillations during a working memory task. Proc Natl Acad Sci USA 100:7931-7936.

Rugg MD, Allan K (2000) Event-related potential studies of long-term memory. In: The oxford handbook of memory (Tulving E, Craik FI, eds), pp 521-538. Oxford: Oxford UP.

Rugg MD, Yonelinas AP (2003) Human recognition memory: a cognitive neuroscience perspective. Trends Cogn Sci 7:313-319.

Rugg MD, Mark RE, Walla P, Schloerscheidt EM, Birch CS, Allan K (1998) Dissociation of the neural correlates of implicit and explicit memory. Nature 392:595-598.
Sobotka S, Diltz MD, Ringo JL (2005) Can delay period activity explain working memory? J Neurophysiol 93:128-136.

Squire LR, Stark CE, Clark RE (2004) The medial temporal lobe. Annu Rev Neurosci 27:279-306.

Tsivilis D, Otten L, Rugg MD (2001) Context effects on the neural correlates of recognition memory: an electrophysiological study. Neuron 31:1-20.

Wilding EL (2005) On the practice of rescaling scalp-recorded event-related potentials. Psychophysiology, in press.

Wilding EL, Rugg MD (1997) An event-related potential study of memory for words spoken aloud or heard. Neuropsychologia 35:1185-1195.

Yonelinas AP (2002) The nature of recollection and familiarity: a review of 30 years of research. J Mem Lang 46:441-517. 